\title{
Multiple-Vehicle Longitudinal Collision Mitigation by Coordinated Brake Control
}

\author{
Xiao-Yun Lu, ${ }^{1}$ Jianqiang Wang, ${ }^{2}$ Shengbo Eben $\mathrm{Li}^{2},{ }^{2}$ and Yang Zheng ${ }^{2}$ \\ ${ }^{1}$ PATH, University of California, Berkeley, CA 94804, USA \\ ${ }^{2}$ The State Key Laboratory of Automotive Safety and Energy, Tsinghua University, Beijing 100084, China \\ Correspondence should be addressed to Jianqiang Wang; wjqlws@tsinghua.edu.cn
}

Received 25 February 2014; Accepted 21 August 2014; Published 28 September 2014

Academic Editor: Valentina Emilia Balas

Copyright (C) 2014 Xiao-Yun Lu et al. This is an open access article distributed under the Creative Commons Attribution License, which permits unrestricted use, distribution, and reproduction in any medium, provided the original work is properly cited.

Rear-end collision often leads to serious casualties and traffic congestion. The consequences are even worse for multiple-vehicle collision. Many previous works focused on collision warning and avoidance strategies of two consecutive vehicles based on onboard sensor detection only. This paper proposes a centralized control strategy for multiple vehicles to minimize the impact of multiplevehicle collision based on vehicle-to-vehicle communication technique. The system is defined as a coupled group of vehicles with wireless communication capability and short following distances. The safety relationship can be represented as lower bound limit on deceleration of the first vehicle and upper bound on maximum deceleration of the last vehicle. The objective is to determine the desired deceleration for each vehicle such that the total impact energy is minimized at each time step. The impact energy is defined as the relative kinetic energy between a consecutive pair of vehicles (approaching only). Model predictive control (MPC) framework is used to formulate the problem to be constrained quadratic programming. Simulations show its effectiveness on collision mitigation. The developed algorithm has the potential to be used for progressive market penetration of connected vehicles in practice.

\section{Introduction}

Road traffic accident is well recognized as one of the major societal problems in the world. In 2009, the numbers of fatalities and injuries were more than 33 thousand and 5.5 million in America and 67 thousand and 238 thousand in China, respectively. Among all accidents, rear-end collisions took up $42.7 \%$ on highway conditions [1]. Many examples of serious crashes can be found online involving over a hundred vehicles $[2,3]$.

Recent years, the market penetration progress of semiautomated vehicles, for example, ACC (adaptive cruise control), has been witnessed. An ACC vehicle relies on onboard radar to detect frontal target vehicles and naturally has throttle control and brake control which could be used for improving safety and reducing workload. Since wireless communication technologies have been well-developed in the past two decades, it provides high possibility to avoid such serious crashes by using simultaneously controlling multiple vehicles, or at least to minimize the impact if the collision is unavoidable. The field operational test (FOT) in [4] suggested that drivers were attracted to using ACC because of (1) the relieved "throttle stress" and (2) the reduction in "headway stress." While it would be beneficial to have ACC simultaneously to consider spacing control, fuel economy, rear-end safety, and driver adaptability, the design of such system becomes rather challenge. One pioneering research was conducted by Li et al. [5]. The main problem for ACC is that if three or more ACC vehicles are in tandem, the system is easy to become string unstable [6]. In fact, a string unstable coupled group of vehicles on highways is more likely to result in multiple-vehicle crashing, which is believed to be the deadly defects of ACC platoon. The concept of cooperative ACC was proposed in [7]. The main advantage of cooperative ACC is the full use of vehicle-to-vehicle communications $(\mathrm{V} 2 \mathrm{~V})$ for information passing to reduce time delay and compensate for remote senor deficiency in control. The string stability for multiple vehicle following for automatic or manual driving was analyzed in [6], which was critical for safety.

For ground vehicles, longitudinal collision warning and mitigation is a long standing topic since it accounts for a large number of collisions on urban freeways. For example, Jansson et al. [8] focused on forward collision by braking and presented a general method for calculating the risk for 
collision by estimating uncertainty and driver maneuvers. The precrash system is another good example for commercial products in many motor companies, which has been developed to avoid/mitigate rear-end collision [9]. This technology uses multiple sensors to detect front vehicles and assists brake operation for reducing the impact in near-collision situation. Honda developed an enhanced collision mitigation system by anticipating the potential collision based on driving conditions, distance to the vehicle ahead, and relative speed [10]. It then used visual and audio warnings to prompt the driver to take preventative action and also to initiate automatic braking if the driver failed to respond. Other similar studies and developments include Volvo [11] and TRL [12]. To reduce actuator delay is critical for collision avoidance/mitigation control algorithms. A design of hydraulic brake assistance system to passenger cars was proposed in $[13,14]$, which directly used master cylinder pressure as the control input. Fang et al. [15] considered the control issue of heavy-duty truck braking system with time delay. The abovementioned technologies can be used as the foundation for implementation of the approach proposed in this paper.

However, many previous works on collision avoidance only took into account two vehicles. This is because the collision avoidance was based on onboard sensor (radar and/or lidar) only without intervehicle communication. The problem for such system was that the last vehicle had to face an accumulated time delay to respond to any maneuver of the vehicles in the front. The objective of this paper is to design a centralized control strategy to avoid longitudinal collision or, if impossible, to mitigate the impact of multiple vehicle longitudinal collision using coordinated brake control. The proposed work is also different from the concept of automated platoon, in which all vehicles are controlled to have automated control capabilities and string stability is major concern in such design. This paper considers the safety improvement using vehicle-to-vehicle communication for current practical highway system by systematically coordinating their braking behaviors. Therefore, it is necessary to consider the relationship between the system and its environment, vehicles in further front and rear, which cannot be coupled into the system without the functionalities of V2V communication and/or automated control capability.

The main contributions of this paper include the following: (a) coupled multiple vehicles are directly taken into account for the formulation of collision avoidance and impact mitigation problem instead of only considering two vehicles as in most previous works; (b) vehicle in further front of the leader vehicle of the coupled group has been taken into account by adding a lower bound as constraint of the deceleration of the leader vehicle; (c) vehicle further behind the coupled group has also been taken into account by adding an upper bound to the deceleration of the last vehicle in the coupled group. This is the typical situation with mixed traffic with progressive market penetration $\mathrm{V} 2 \mathrm{~V}$ and automated vehicle control capabilities.

The remainder of this paper is organized as follows: Section 2 is the Nomenclature section; Section 3 presents system analysis and mathematical modeling; Section 4 is devoted to MPC design based on vehicle following model;
Section 5 is simulation scenario runs and analysis; Section 6 is for concluding remarks.

\section{Nomenclature}

The following notations will be used throughout the paper:

$m_{n}$ : Mass of vehicle $n$

$L_{n}$ : Length of vehicle $n$

$d_{n}(t)$ : Brake deceleration of vehicle $n$

$x_{n}(t)$ : Longitudinal distance of vehicle $n$ with respect to a common inertia coordinate system

$s_{n-1, n}(t)$ : Intervehicle distance between vehicle $n-1$ and vehicle $n$

$v_{n}(t)$ : Longitudinal speed of vehicle $n$

$v_{n-1, n}(t)$ : Relative speed between vehicle $n-1$ and vehicle $n$

$t_{0}$ : System braking start time

$x_{n}^{0}$ : Longitudinal distance of vehicle $n$ with respect to a common inertia coordinate system at time $t_{0}$

$v_{n}^{0}$ : Longitudinal speed of vehicle $n$ at time $t_{0}$

$D_{n}^{\max }$ : Brake deceleration capability of vehicle $n$

$c_{1}$ : The lower bound limit on deceleration of the first vehicle

$c_{N}$ : The upper bound on maximum deceleration of the last vehicle

$T_{d}$ : Driver reaction time

$T$ : Time interval for system discretization

$N$ : The number of vehicles in the longitudinal coupled group

$N_{p}$ : Length of predictive horizon in MPC

$N_{c}$ : Length of control horizon in MPC.

The subscript $n(n \in\{1,2, \ldots, N\})$ is the vehicle index in the longitudinally coupled group and $n=1$ is the first (leading) vehicle. Further, we have

$$
\begin{gathered}
s_{n-1, n}(t)=x_{n-1}(t)-x_{n}(t), \\
v_{n-1, n}(t)=v_{n-1}(t)-v_{n}(t) .
\end{gathered}
$$

\section{System Analysis and Modeling}

To consider the collision avoidance and impact mitigation for the scenario of multiple-vehicle collision, it is only necessary to consider a system composed of closely following vehicles, longitudinally coupled. Two adjacent vehicles in the same lane are considered coupled if their speed and distance (or time headway) jointly satisfy some conditions. Intuitively, this can be stated as follows: if any longitudinal maneuver of the subject vehicle would require its follower to take immediate action to ensure safety (to avoid collision), those vehicles are considered coupled. For natural highway driving, the time headway is usually $0.7 \sim 3.8 \mathrm{~s}$ [16]. Therefore, most vehicles in the same lane are considered coupled in some group in highway traffic. 
How to define the boundary between coupled group? This will depend on several factors including (1) the type of vehicles involved; (2) the maximum deceleration capability; (3) current speed and following distance (or time headway); (4) practical tire slip; (5) road geometry; and (6) wireless communication availability. The tire slip will be considered as the external disturbance to the speed and distance measurement later. It is clear that wireless communication is the critical factor for the coordinated control of brakes among a platoon of coupled vehicles, with which the vehicle types and deceleration capability can be broadcasted in the message set. To make the algorithms adapt to progressive market penetration of $\mathrm{V} 2 \mathrm{~V}$, the system boundaries also include those points where the vehicles do not have wireless capability.

The overall control system for the group of coupled vehicles can be divided into two parts: upper level control and lower level control (Figure 1). The function of upper level control is to generate desired deceleration for each vehicle based on system states (relative position and speed) to avoid collision or to minimize potential impact (if collision is unavoidable) subjected to the constraints of deceleration capabilities for each vehicle. The lower level control is on individual vehicle, which activates the brake control to achieve the desired deceleration. This work will focus on the upper level control.

From a practical viewpoint, each vehicle may have a different powertrain and control system depending on the type of the vehicle. As an example, many passenger cars are equipped with disk brake which may be hydraulic or electric, and other vehicles may also have hydraulic brake drums; and most heavy-duty vehicles are equipped with brake including three subsystems: pneumatic brake, engine retarder (Jake brake), and transmission retarder. The differences between vehicle types in a coupled group are quantified in this paper as the deceleration capability (of braking system). Such characterization is for the simplicity of modeling. The maximum deceleration capability could be reasonably considered nearly constant for most of the speed profile of a vehicle.

For simplicity of discussion, the collision impact mitigation system is only activated if certain collision threat threshold has been reached [17]. This can be determined by sensor detection, threat assessment, and warning to the driver. If any driver does not respond appropriately, the coordinated brake control for each vehicle will be activated as the last resort.

3.1. Overall System Structure. The overall system structure for multiple-vehicle collision avoidance and impact mitigation could be depicted in Figure 1. It includes remote sensor detection of relative distance and speed detection/estimation, V2V (DSRC), control, and decision making.

3.2. The System and Critical Time Points. Look-ahead time or time to collision (T2C) is very important to vehicle following manually or by automatic control. It is suggested that the $\mathrm{T} 2 \mathrm{C}$ is refined into the following time point sequences for improved performance in active vehicle safety (Figure 2).

(i) System coupling time: if any of the intervehicle distance of a group of vehicles in the same lane is within a specified threshold with potential collision judged

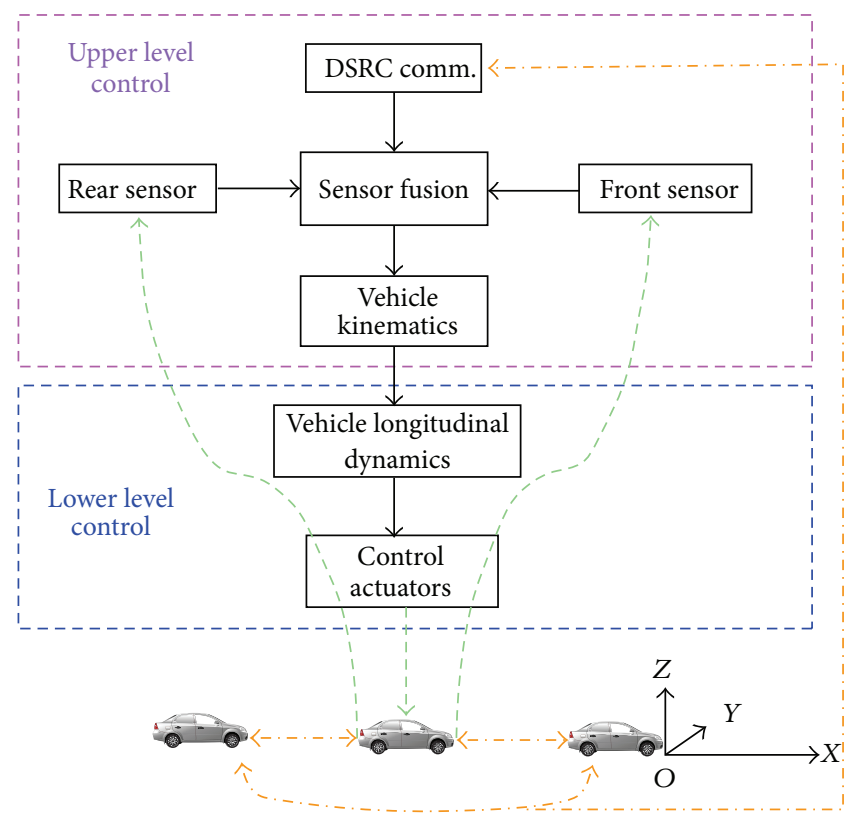

FIGURE 1: Overall picture the two-layer control system for multiplevehicle collision avoidance and impact mitigation.

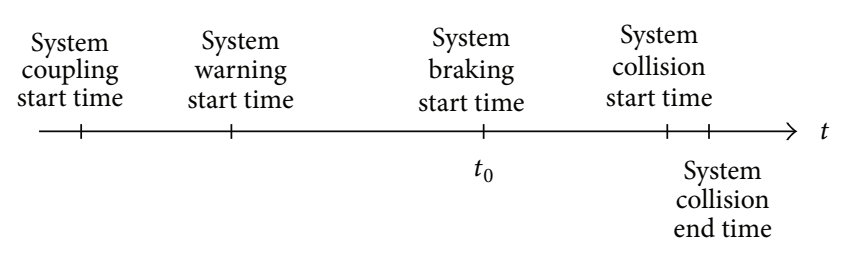

FIGURE 2: Sequential time instant for active vehicle collision warning and avoidance analysis.

by some threat assessment criteria [17] persistently for certain time period, the group of vehicles are called coupled. The corresponding time is the coupling time. Outside this threshold, the consecutive vehicles are considered decoupled or independent. In this paper, the V2V capability of all the vehicles in the coupled group for coordinated control is further assumed.

(ii) System warning time: warning signal is activated to the driver to act for collision avoidance (passive).

(iii) System braking start time $t_{0}$ : it is coordinated brake control actuation start time which is necessary to avoid collision if driver does not respond to the warning correctly, or if not possible, the earliest time possible to activate the brake for collision impact mitigation (active).

(iv) System collision start time: it is time instant for at least one pair of vehicles to start collision.

(v) System collision end time: it is time instant for the collision scenario ending.

The active control algorithm developed in this paper is for the time period starting from $t_{0}$ as indicated in Figure 2.

It is noted that the magnitude of each period shown here needs to be determined quantitatively in a scientific 
way. In this paper, we only focus on the control action by braking starting from $t_{0}$ to the time point when the maneuver (collision avoidance or impact mitigation) is finished. It is clear that only coupled vehicles need to be taken into account in the system.

3.3. Upper Level Control Strategy. Considering the differences between vehicles involved in the following aspects, mass, deceleration capability (depending on braking capability, tire slip, and road surface condition), sensor detection, control actuation, and overall time delay, it is impractical to apply a constant deceleration for each vehicle. Instead, the braking torque of each vehicle needs to be adjusted according to current and predicted states of the system based on sensor measurements and previous information. The system states include the relative distance and speed of each vehicle. An upper level control strategy could be stated as follows.

(1) First vehicle should brake as less as possible to fully use the space in the front and to minimize impact with its follower. However, this is limited by the presence/absence of vehicle in its front.

(2) The last vehicle should brake as hard as possible to the extent to fully use its rear space and to minimize its impact to its front vehicle. Similarly, this may be limited by the presence of vehicle in its rear.

(3) The desired deceleration between all the other vehicles will be determined by the current and predicted state of each vehicle, which is estimated based on realtime sensor measurement.

(4) The prediction for the state in finite time horizon of each vehicle is conducted by a model predicted approach.

In summary, an algorithm for frontal-rear collision avoidance and impact minimization should take the following factors into account:

(i) multiple-vehicle collision scenario,

(ii) mechanical definition of impact,

(iii) vehicle mass,

(iv) relative distance and relative speed at braking,

(v) relative distance and speed at collision (time instant of impact),

(vi) deceleration capability (braking capability) of each vehicle in the coupled group,

(vii) constraint to the first vehicle in the front end of the coupled group,

(viii) constraint to the last vehicle in the rear end of the coupled group.

3.4. Assumptions. Based on vehicle design and the effect of front and rear bumpers, the collision in consideration is neither perfect elastic nor perfect inelastic. A quantitative description of the multiple-vehicle collision scenario also depends on the type of vehicles. In those cases, the kinetic energy during collision is not conserved. Besides, it would be difficult to accurately estimate the collision impact for each pair of vehicles. Therefore, we use the relative kinetic energy before collision as the measure of the potential impact in collision of a pair of vehicles, which in turn is used to estimate the overall system potential impact. This is physically justified since the collision is the work done by the relative kinetic energy. To properly define the system, The following is further assumed.

Assumption 1. The minimum deceleration of the first vehicle and the maximum deceleration of the last vehicle in the coupled group are known (relationship with environment), for avoiding colliding with the vehicles in the front and the end of the coupled group.

Assumption 2. Sensor detection: each vehicle has a frontlooking and rear-looking radar/lidar to detect intervehicle distance and relative speed reliably. Such information can be used as redundancy for reliability and time delay reduction of sensor measurement within the system, which is necessary for determining the relationship with vehicles outside of the system.

Assumption 3. Intervehicle communication systems (V2V): any pair of vehicles in the coupled group can communicate with each other (2-way communication).

A kinematic vehicle model based on a common inertia coordination system on the ground under the front bumper of the first vehicle is

$$
\begin{gathered}
\dot{x}_{n}(t)=v_{n}(t), \\
\dot{v}_{n}(t)=-d_{n}(t), \\
\dot{s}_{n-1, n}(t)=v_{n-1, n}(t), \\
\dot{v}_{n-1, n}(t)=d_{n}(t)-d_{n-1}(t), \\
s_{n-1, n}\left(t_{0}\right)=s_{n-1, n}^{0}, \\
v_{n-1, n}\left(t_{0}\right)=v_{n-1, n}^{0}, \\
n=2, \ldots, N .
\end{gathered}
$$

It is noted that the initial condition of relative distance and relative speed at the time instant $t_{0}$ are known, but after that time point, they are determined by the control processes and system responses. However, they can be estimated based on sensor measurement and predicted by system model in a MPC process.

3.5. Objective Function. Although there are several alternatives, it is suggested to adopt the following total relative kinetic energy as the objective function:

$$
J=\frac{1}{2} \sum_{n=2}^{N} m_{n} v_{n-1, n}^{2}(t) .
$$

In practice, the real impact only happens during the collision. The collision impact should be taken into account only for the case $v_{n-1, n}(t) \leq 0$. 


\section{MPC Control Formulation}

By defining $f(k):=f\left(t_{0}+k T\right), k=0,1, \ldots$, for a function $f(\cdot)$, the system can be discretized as follows for control design:

$$
\begin{gathered}
x_{n}(k+1)=x_{n}(k)+v_{n}(k) T \\
v_{n}(k+1)=v_{n}(k)-d_{n}(k) T \\
x_{n}(0)=x_{n}^{0} \\
v_{n, n+1}(0)=v_{n}^{0} \\
n=1, \ldots, N .
\end{gathered}
$$

The objective function and the constraints over the predicted time horizon are expressed as

$$
\begin{aligned}
& J(k)=\frac{1}{2} \sum_{j=1}^{N_{p}} \sum_{n=2}^{N} m_{n} v_{n-1, n}^{2}(k+j) \\
& \min _{\mathbf{d}} J(k) \\
& \text { subject to }\left\{\begin{array}{l}
0 \leq d_{n}(k+j) \leq D_{n}^{\max } \\
d_{1}(k+j) \geq c_{1}>0 \\
d_{N}(k+j) \leq c_{N}
\end{array}\right. \\
& n=1, \ldots, N ; \quad j=0, \ldots, N_{c}-1, \\
& x_{n-1}(k+j)-x_{n}(k+j)>L_{n-1}, \\
& n=2, \ldots, N ; \quad j=1, \ldots, N_{p} .
\end{aligned}
$$

The solution to the optimization problem (5) is the sequence of input signals $\mathbf{d}=[\mathbf{d}(k), \mathbf{d}(k+1), \ldots$, $\left.\mathbf{d}\left(k+N_{c}-1\right)\right]^{T}$, where $\mathbf{d}(k+j)=\left[d_{1}(k+j), d_{2}(k+j), \ldots\right.$, $\left.d_{n}(k+j)\right]^{T}$. At each time step, $J(k)$ is minimized to determine the brake control for each vehicle while subjecting to the constraints of the system dynamics (4). The second constraint in (5) is necessary. One can easily create a similar example to show the necessity of the third constraint. The constraint used in (5) can be refined for the following scenarios.

Scenario 0: the default situation: for the coupled group, there is a vehicle in the front and a vehicle behind; both do not have V2V or control capability. In this case, all the constraints in (5) need to be taken into account in MPC at each time step.

Scenario 1: the vehicle in front of the coupled group is far enough and there is no other object in the way. Therefore the leading vehicle can choose not to brake at all. In this case, the second constraint in (5) $d_{1} \geq c_{1}>0$ can be dropped.

Scenario 2: the vehicle following the last vehicle is far enough without any threat to the coupled group from behind. Therefore the last vehicle in the platoon could brake as hard as possible. In this case, the third constraint in (5) $d_{N} \leq c_{N}$ should be ignored.

Scenario 3: there is no vehicle in the front or behind. In this case, both $d_{1} \geq c_{1}>0$ and $d_{N} \leq c_{N}$ should be ignored.

It is expected that, for Scenario 3, if the initial speed for each vehicle at the time of coordinated brake is very close and if each vehicle adopts the desired deceleration as the minimum of the deceleration capabilities of all the vehicles involved, collision would likely be avoided. This situation is therefore trivial which agrees with the simulation results observed.

\section{Simulation}

Since the algorithm is designed for practical coordination and control for multiple-vehicle longitudinal collision avoidance and impact mitigation, it should allow different vehicle types in a coupled group. However, the number of vehicles is known as a priori. The difference in vehicle types is characterized by the following parameters: vehicle mass, length, and deceleration capability. In order to verify the proposed coordinated brake control performance, the other three strategies, direct braking control, driver-reaction based brake control, and LQR control are built for simulation. These four methods are defined as follows.

(i) Coordinated Brake Control (CBC). This method coordinates the deceleration for each vehicle using MPC, which is formulated into a constrained quadratic programming. The design details of $\mathrm{CBC}$ can be found in Sections 3 and 4.

(ii) Driver Brake Control (DBC). This method has the same assumption and condition that $\mathrm{CBC}$ faces. The vehicles in the coupled group should have the V2V communication ability and the ability to automatically brake. The difference between $\mathrm{DBC}$ and $\mathrm{CBC}$ is that $\mathrm{DBC}$ directly employs the full decelerating ability for each vehicle rather than coordinating the deceleration for each vehicle when some emergency situation happens. It means that each vehicle in the coupled group immediately hits the brakes fully when facing some critical accidents.

(iii) Driver-Reaction Based Brake Control (DRBC). This method does not assume that the vehicles in the coupled group have the V2V communication ability and automatically braking ability. The driver judges the driving situation and brakes the vehicle fully after certain driver reaction time when emergency situation happens.

(iv) Linear Quadratic Regulator Control (LQR). This method employs the linear quadratic regulator (LQR) control during the braking process, which is a classical optimal control strategy for cooperative adaptive cruise control (CACC) during normal driving situation. In this paper, the constant time headway policy is used for the car-following model, and the control objective is to follow driver's desired spacing (see [18] for more details).

5.1. Simulation Development. For most cases, vehicles with large mass will be longer and will have lower deceleration capability. For this reason, once the number of vehicles is specified in simulation, the mass for each vehicle is generated randomly. Then the vehicle length and deceleration capability are determined according to the following rule. The minimum mass used is $M_{\min }=1000 \mathrm{~kg}$ (passenger car), and maximum mass is $M_{\max }=15000 \mathrm{~kg}$ as half-loaded heavyduty trucks. For given number of vehicles $N$, the mass of each vehicle is randomly generated as follows:

$$
\begin{array}{r}
m_{n}=M_{\text {min }}+\left(M_{\text {max }}-M_{\text {min }}\right) \cdot \operatorname{rand}(1) \\
n=1, \ldots, N .
\end{array}
$$



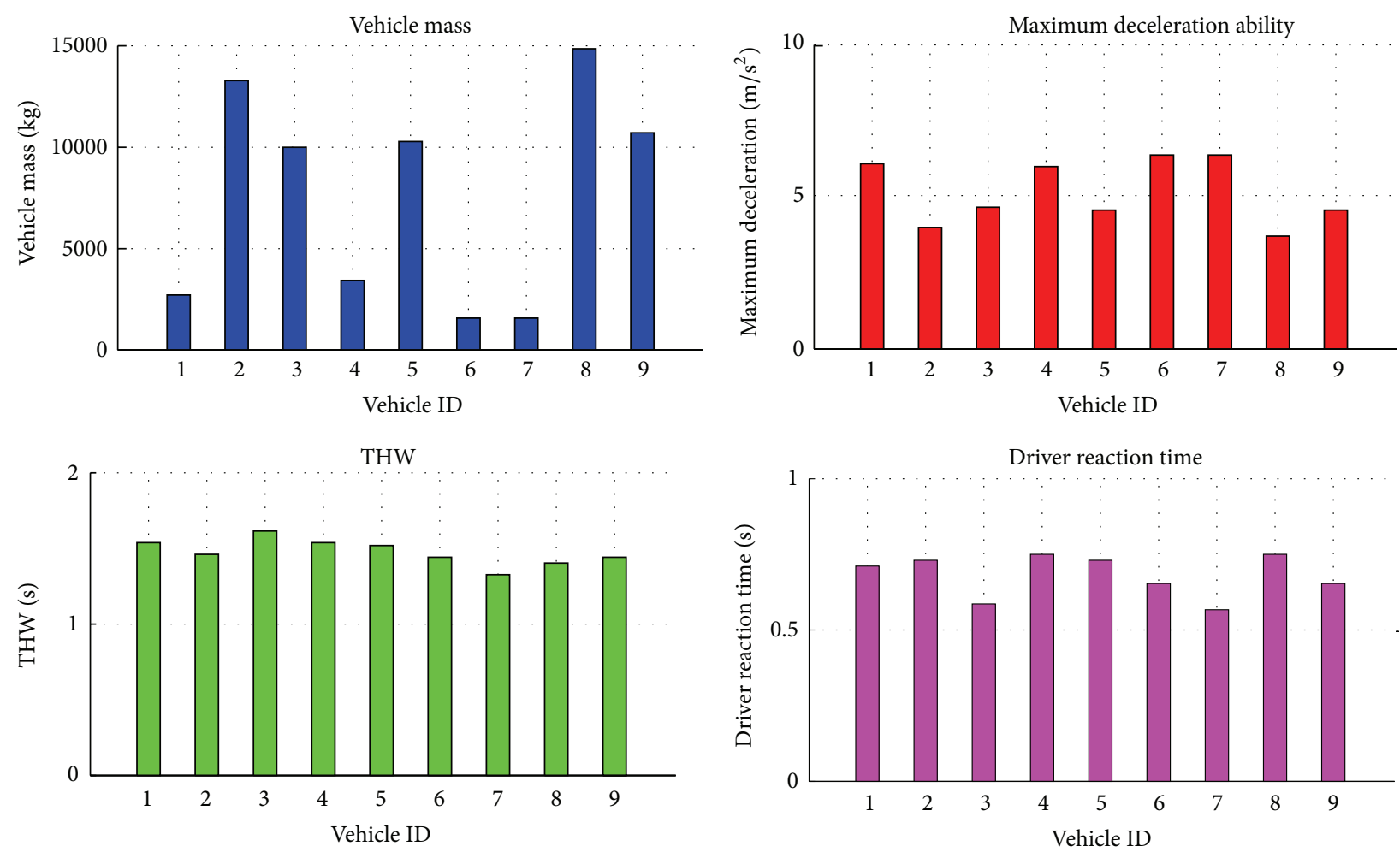

FIGURE 3: Randomly generated vehicle mass, maximum deceleration, THW, and driver reaction time; abscissa: vehicle ID.

With the generated mass, the vehicle length is determined as follows:

$$
L_{n}=3.0(1-\alpha)+23.0 \alpha, \quad \alpha=\frac{m_{n}-M_{\min }}{M_{\max }},
$$

which means that the minimum vehicle length is $3.0 \mathrm{~m}$ (passenger car) and maximum vehicle length is $23 \mathrm{~m}$ (tractor trailer combination). Vehicle deceleration capability is also generated based on masses as follows:

$$
D_{n}^{\max }=3.0\left(2.2-\frac{m_{n}}{M_{\max }}\right) .
$$

According to this formula, for a $1000 \mathrm{~kg}$ passenger car, the maximum deceleration is $6.4 \mathrm{~m} / \mathrm{s}^{2}$, while it is about $3.6 \mathrm{~m} / \mathrm{s}^{2}$ for a $15000 \mathrm{~kg}$ truck. Employing time headway (THW) policy, we can get the car following distance distribution in the coupled group. As [16] points out, THW is usually from $0.7 \mathrm{~s}$ to $3.8 \mathrm{~s}$ in Chinese highway. In this paper, we make the THW distribution follow Gaussian distributions THW $N\left(1.5,0.1^{2}\right)$. The work in [19] points out that the driver reaction time for warning signal is spread over the period from $0.3 \mathrm{~s}$ to $2 \mathrm{~s}$ and the average driver reaction time is $0.66 \mathrm{~s}$. We make the driver reaction time obey the Gaussian distribution $T_{d} \sim N\left(0.66,0.1^{2}\right)$ for our simulations.

5.2. Simulation Results. In this section, we present the typical scenario simulation results using the four control strategies. We also run 100 simulations with certain random parameters and the statistical results are presented below. All the simulation scenarios involve nine vehicles. The predictive horizon and control horizon are all 5 steps, and the length of time step used for simulation is $0.02 \mathrm{~s}$. The initial speed for each vehicle is $31 \mathrm{~m} / \mathrm{s}$ plus $\pm 10 \%$ random fluctuation.

5.2.1. Typical Scenario Simulation. The minimum deceleration of the leading vehicle is limited to its maximum deceleration capability, which means that the vehicle in the front of coupled group is braking so that the first vehicle in the coupled group needs braking to avoid the accident. The maximum deceleration of the last vehicle is limited to its maximum deceleration capability, which means that there is no vehicle following the last vehicle in the coupled group and the last vehicle in the coupled group can brake as hard as possible to avoid collision with the vehicle ahead. The mass, deceleration capability, THW, and driver reaction time distribution for the coupled group are shown in Figure 3. With initial speed for each vehicle and THW for each driver, the initial spaces between vehicles are determined, which can be obtained through distance sensors, like radar/lidar or video process in real implementation.

Vehicle trajectories and speed profiles for the coupled group produced by CBC are illustrated in Figures 4 and 5 . The input sequence (desired deceleration profiles) for each vehicle, which is generated by MPC method, is demonstrated in Figure 6. The simulation results show collision avoidance of all the vehicles. After about 9 seconds, all the vehicles stopped by coordinated brake control. It can be observed from Figure 4 that all the intervehicle distances remain to be greater 


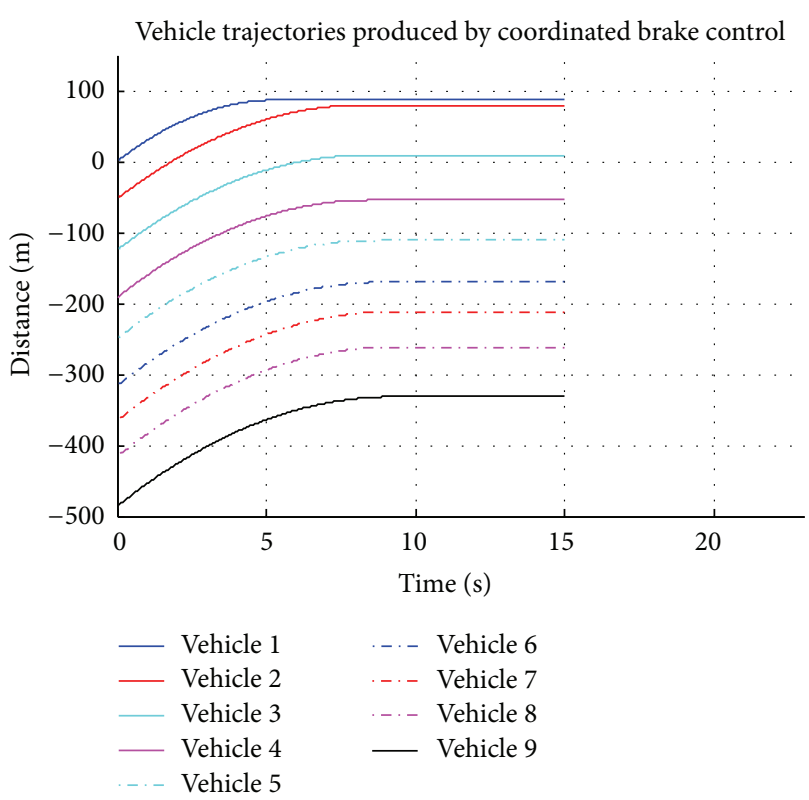

FIGURE 4: Nine vehicle trajectories produced by CBC.

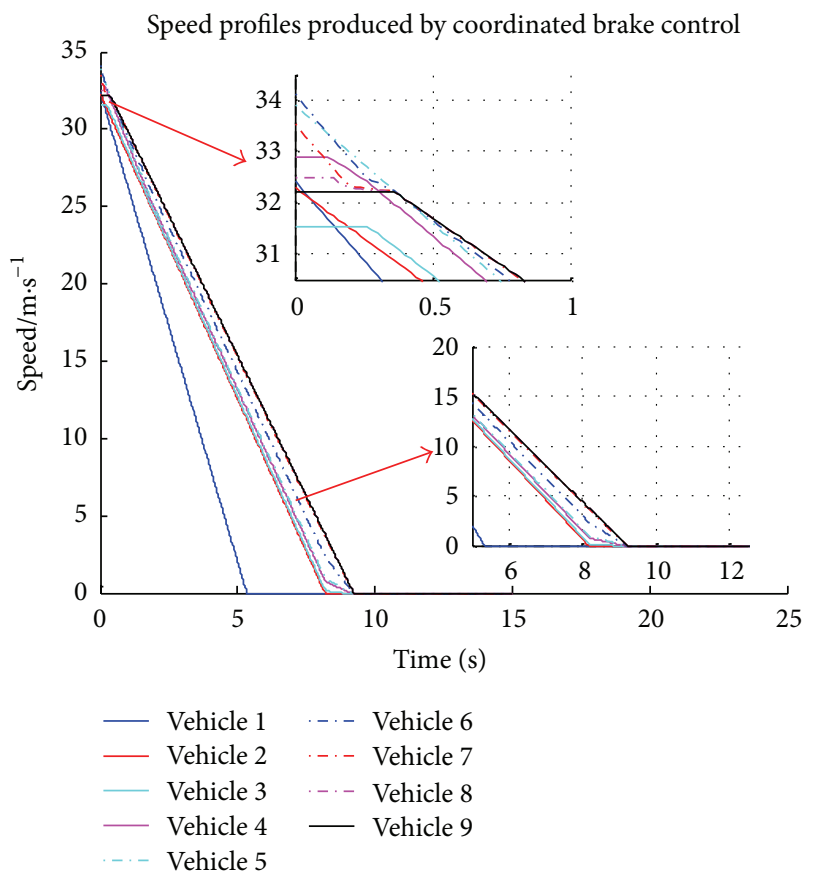

Figure 5: Nine vehicle speed profiles produced by CBC.

than zero during the braking process, which means that crash has been completely avoided in the group. As stated above, the vehicles in further front of and behind the system are represented by the constraints of the minimum deceleration of the leading vehicle and the maximum deceleration of the last vehicle in the coupled group. Simulation results demonstrated that coordinated brake control can not only regulate the relative speed within a reasonable range but also keep the space between vehicles within a reasonable range.

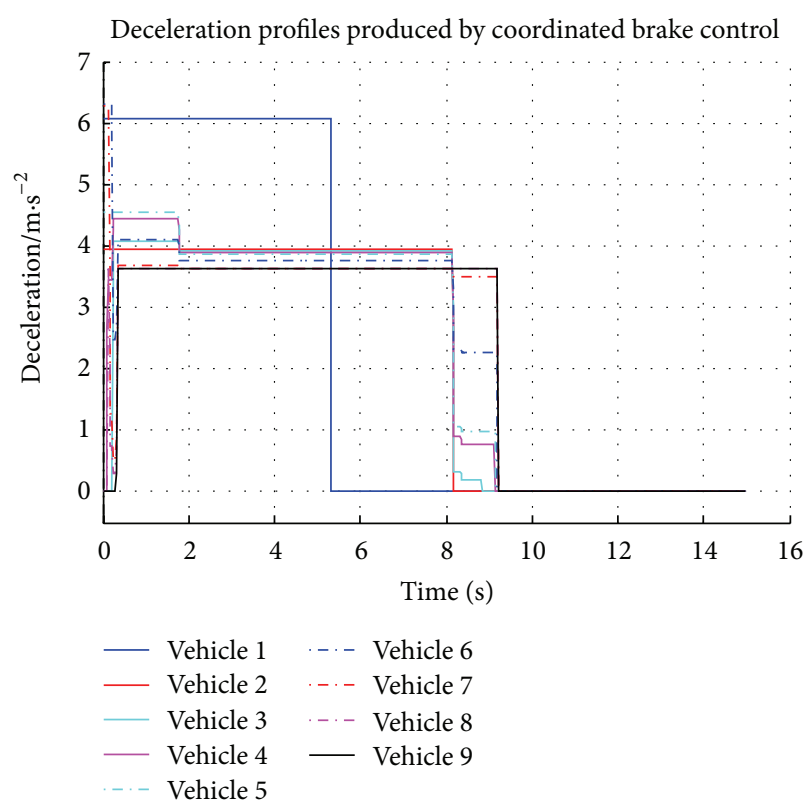

Figure 6: Nine vehicle desired deceleration profiles produced by CBC.

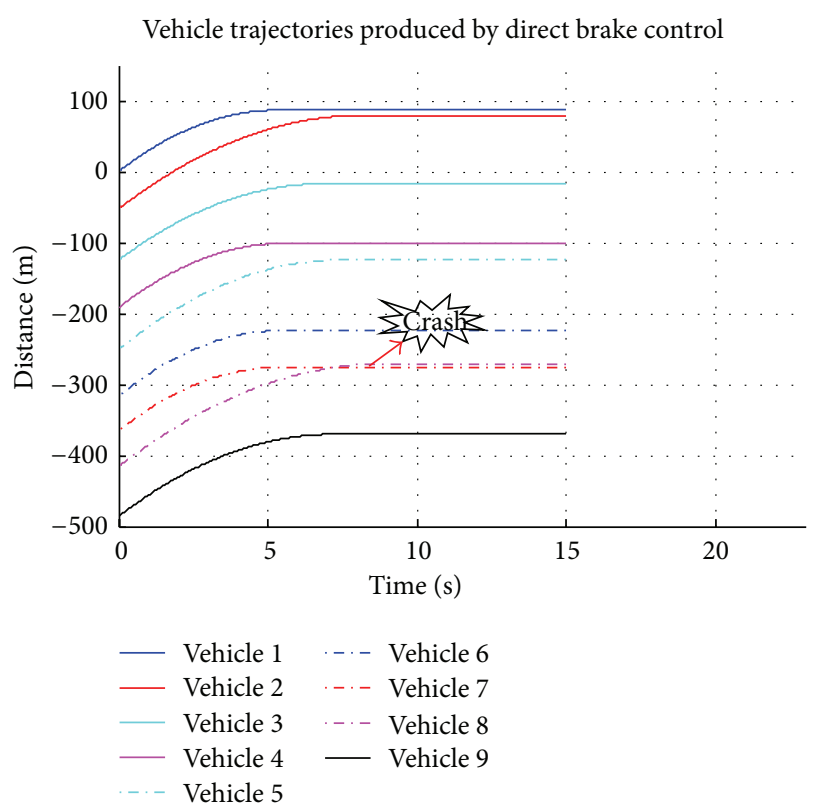

FIGURE 7: Nine vehicle trajectories produced by DBC.

Although the initial speeds of each vehicle in the coupled group appear to be random, the vehicle speed becomes regular under coordinated brake control after several seconds, as shown in Figure 5. The optimization function (3) is to minimize the total relative kinetic energy in the coupled group, which is equivalent to keep all vehicle speed as close as possible. If all the vehicles are identical, which means that the vehicle has same deceleration ability for our problem, the vehicles' speed can be controlled into no difference. To some degree, this situation means that the vehicles in the coupled 


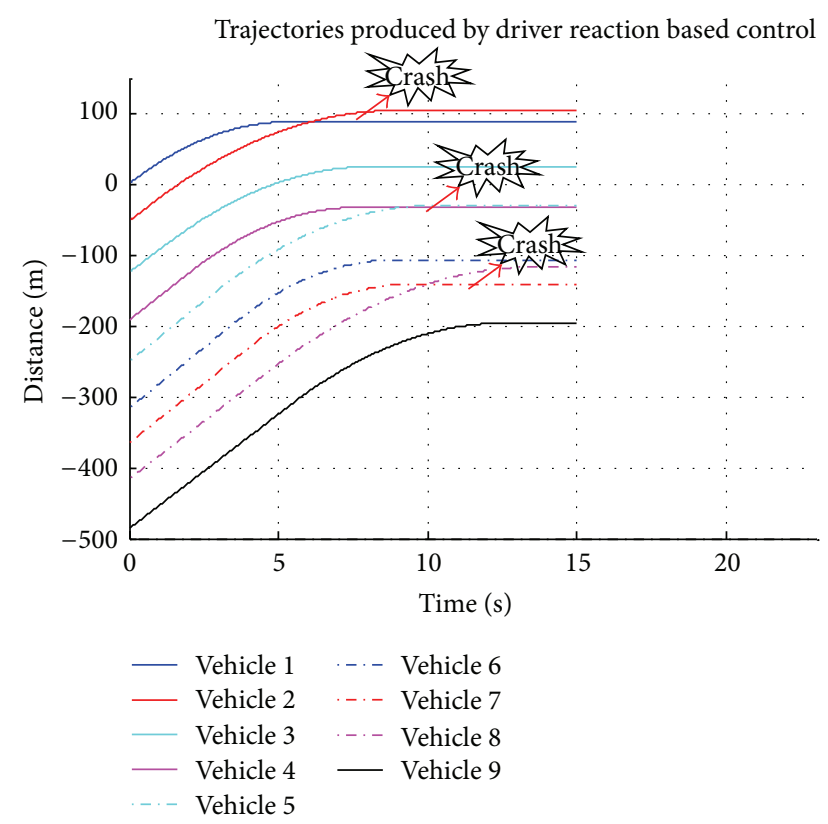

FIGURE 8: Nine vehicle speed profiles produced by DRBC.

group can be treated as one vehicle, whose length is enlarged. However, the deceleration capability is usually inconsistent, as demonstrated in Figure 3 for our simulation.

For the same condition, if we use DBC, DRBC, or LQR control, we can get simulation results shown in Figure 7 to Figure 9. One point to note is that the leading vehicle in the coupled group brakes fully for these three simulations. Figure 7 demonstrates the vehicle trajectories produced by DBC, and the vehicle trajectories generated by driver reaction based control are shown in Figure 8. Figure 9 demonstrates the vehicle trajectories produced by LQR.

It can be observed that there is one rear-end accident which happened between vehicle 7 and vehicle 8 for DBC in Figure 7. Vehicle 7 has good deceleration capability $\left(D_{7}^{\max }=6.29 \mathrm{~m} / \mathrm{s}^{2}\right)$ and vehicle 7 is stopped within 90 meters. However, vehicle 8 has poor deceleration capability $\left(D_{8}^{\max }=\right.$ $3.63 \mathrm{~m} / \mathrm{s}^{2}$ ) and vehicle 8 needs over 140 meters to stop completely. The difference between these two stop distances is larger than the car following distance, which is determined by THW. So rear-end collision happens between these two vehicles. As shown in Figure 8, there are three crash accidents which happened (vehicle 1 and vehicle 2, vehicle 3 and vehicle 4 , and vehicle 7 and vehicle 8 ) for DRBC. The difference of deceleration capability between vehicles is the cause of crash accident, the same analysis as DBC. Another main cause is the driver reaction time to emergency incident. It is the second driver's response time that causes the rear-end collision between vehicle 1 and vehicle 2 , which can be avoided if the response time is eliminated as DBC performance. As shown in Figure 9, there is also one collision accident which happened between vehicle 1 and vehicle 2 for LQR control.

Figure 10 shows the comparison of relative kinetic energy produced by four brake control strategies during the brake

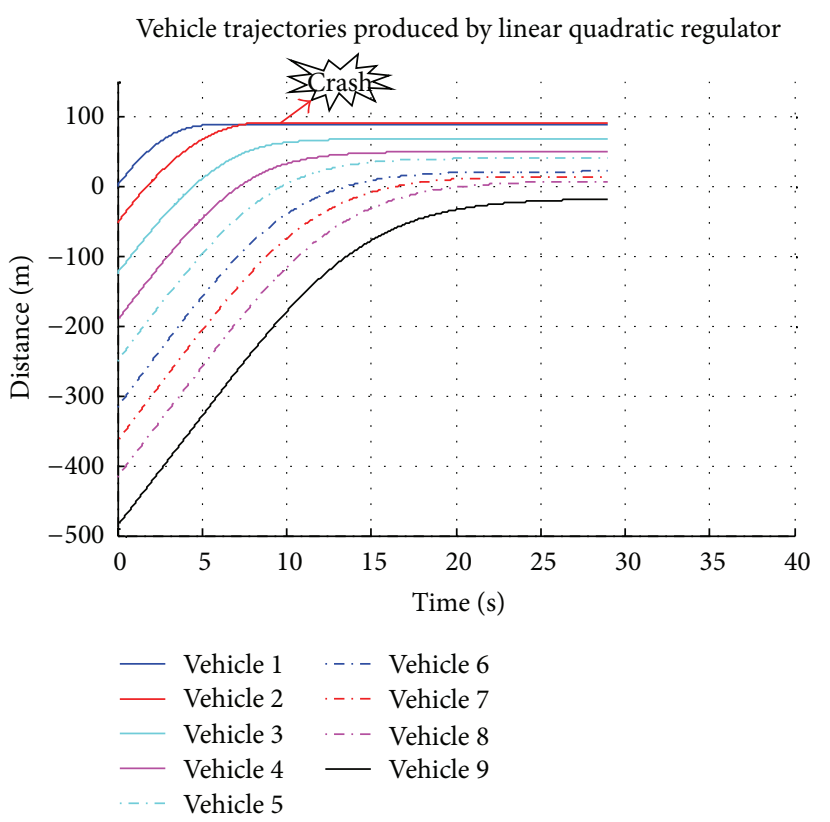

FIGURE 9: Scenario 1: nine vehicle speed profiles produced by LQR control.

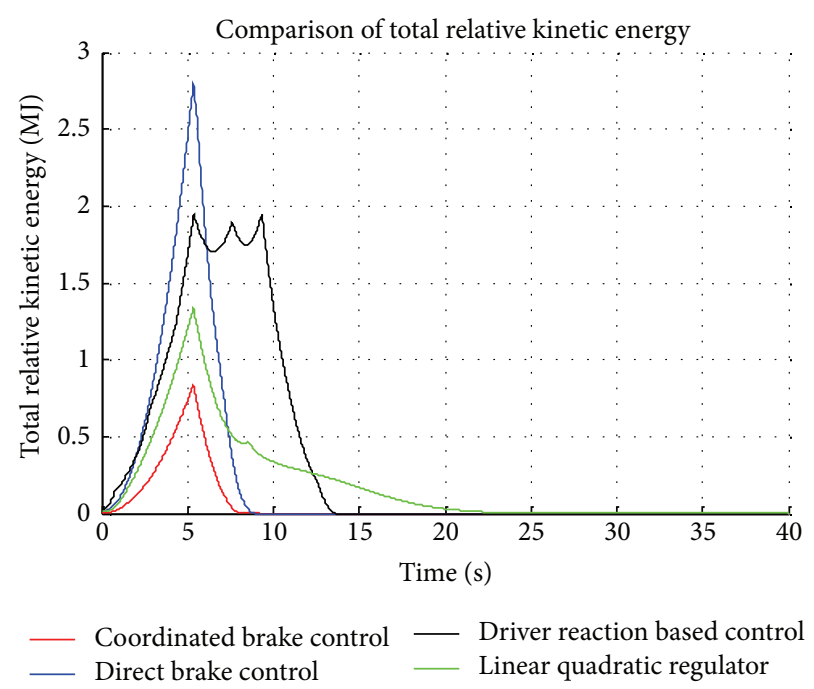

FIGURE 10: Comparison of total relative kinetic energy produced by four control strategies.

process. One point to be noted is that this figure is only schematic for we disregard the collision.

5.2.2. Multisimulation Result. In order to test the proposed CBC performance under different parameters, we run 100 simulations with certain random generated parameters.

Figure 11 shows the vehicle attribute (mass, length, and maximum deceleration capability) distribution for these 100 simulations. In order to make the situation more hazard, we add one small vehicle, whose mass is generated from $1000 \mathrm{~kg}$ to $3000 \mathrm{~kg}$ randomly, and one large vehicle, whose mass is generated from $1000 \mathrm{~kg}$ to $15000 \mathrm{~kg}$ randomly in each 

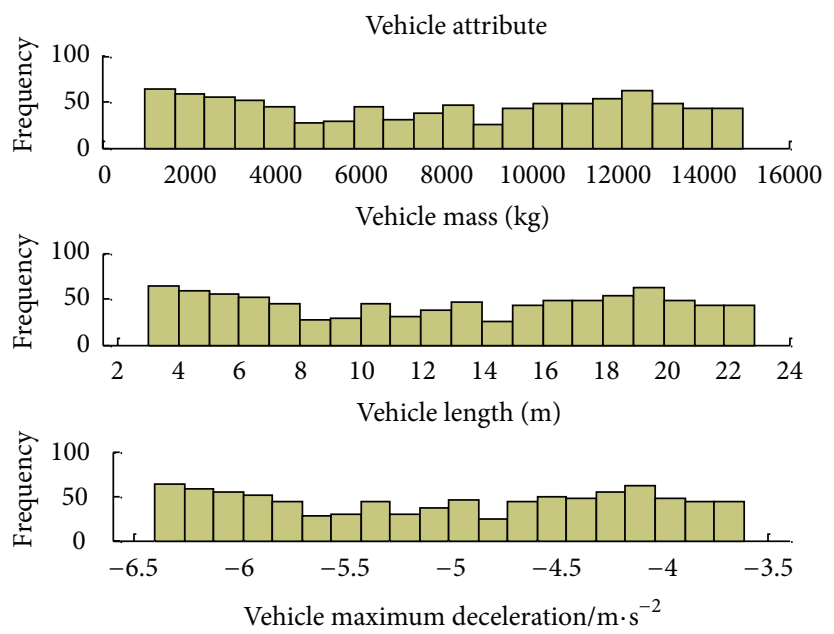

FIGURE 11: Vehicle attribute (mass, length, and maximum deceleration) distribution for 100 simulations.
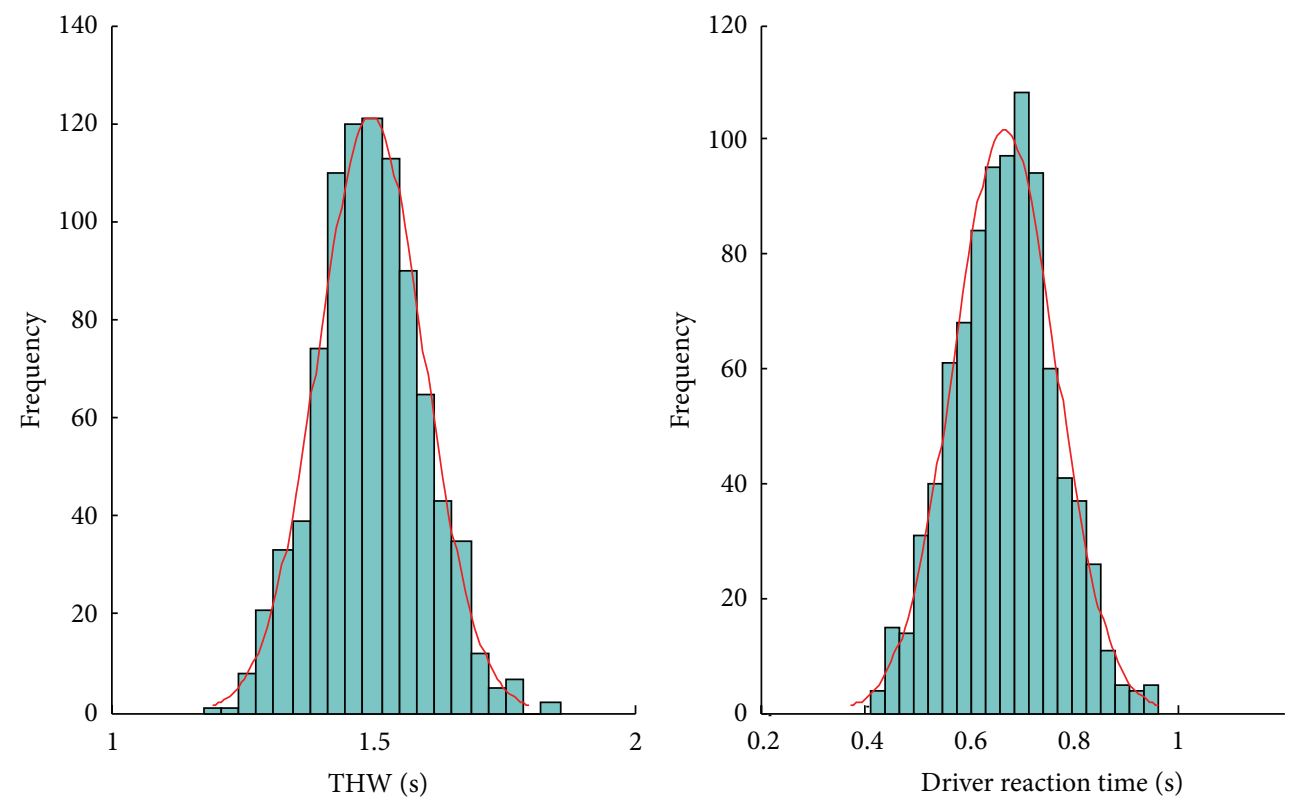

FIGURE 12: THW and driver reaction time distribution for 100 simulations.

simulation. The positions of these two vehicles are placed in the coupled group randomly and guarantee the large vehicle is at the back of the little vehicle. Figure 12 shows the distributions of THW and driver reaction time, which are generated by formulas $T_{d} \sim N\left(0.66,0.1^{2}\right)$ and $T_{d} \sim$ $N\left(0.66,0.1^{2}\right)$, respectively. The initial speed for each vehicle is $31 \mathrm{~m} / \mathrm{s}$ plus random fluctuation.

Figure 13 shows the successful rate for collision avoidance using different brake control strategies. During these 100 simulations, there are 95 cases that avoid collision successfully when using $\mathrm{CBC}$. When switching to $\mathrm{DBC}, \mathrm{DRBC}$, and LQR control, the successful rate is reduced from $95 \%$ to $81 \%$, $14 \%$, and $84 \%$, respectively. CBC has the best performance for collision avoidance among these four control strategies. On the other hand, LQR for CACC have good performance under normal situation and even have string stability when satisfying some conditions [18]. However, LQR may not have good performance for collision avoidance under emergency situation, as shown in Figure 13.

Figure 14 shows the box plot of relative kinetic energy and relative speed when two vehicles collide with each other using four different control strategies. Simulation statistics shows that $\mathrm{CBC}$ has best collision impact mitigation for these four control strategies, for the collision impact (relative kinetic energy) is usually the minimum among these four control strategies. Figure 15 shows the box plot of statistical data of brake successful cases using four different control strategies. Figure 16 shows the collision vehicle type. Most collision happens between one large vehicle with poor deceleration ability and one small vehicle with good deceleration ability. Under this condition, the collision usually cannot be avoided no matter which method is employed. A possible solution 


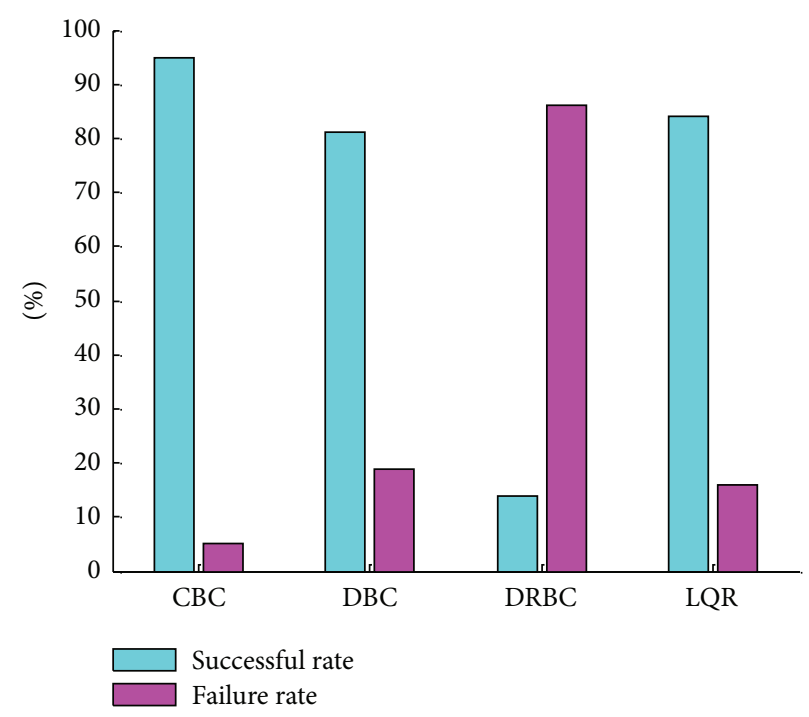

FIGURE 13: Successful rate and failure rate for four control strategies.
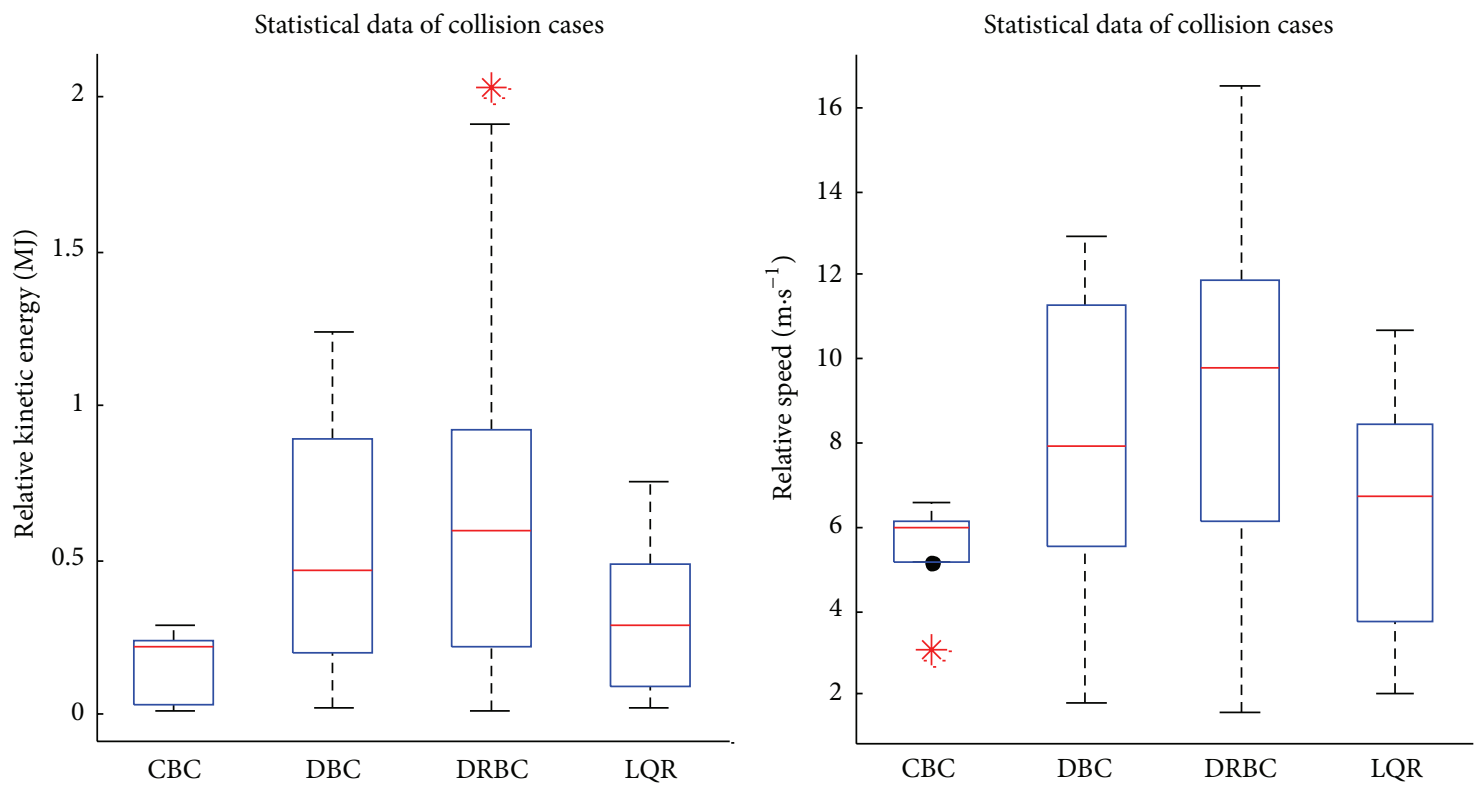

FIGURE 14: Relative kinetic energy and relative speed distributions for collision cases under four different control strategies (CBC: 5 cases; DBC: 19 cases; DRBC: 84 cases; LQR: 16 cases).

is to enlarge the car following distance for large vehicle or reduce the driving speed. Driver reaction time is another essential factor for vehicle collision. It can be observed that the scatter of vehicle type for DRBC is the most disperse from Figure 16. Although $\mathrm{CBC}$ has best performance in general, collision may happen between two small vehicles as Figure 16 demonstrated.

\section{Concluding Remarks}

Upper level control to avoid or to minimize the impact of multiple-vehicle longitudinal collision has been considered in this paper. The objective is to generate desired deceleration for each vehicle in the coupled group. To implement this concept would require all the vehicles in the coupled group be wirelessly connected. In practice, market penetration of vehicles with wireless and frontal and rear sensor (radar/lidar) is a progressive process. However, the approach in this paper should be used in any stage: only taken into account those consecutive vehicles in the same lane with those capabilities in coupled group. A kinematic model is used to describe the dynamic relationships between vehicles in the coupled group. Those constraints also take into account the vehicle in front and rear of the group which cannot be coupled due to the lack of wireless and control capabilities. All the possible 

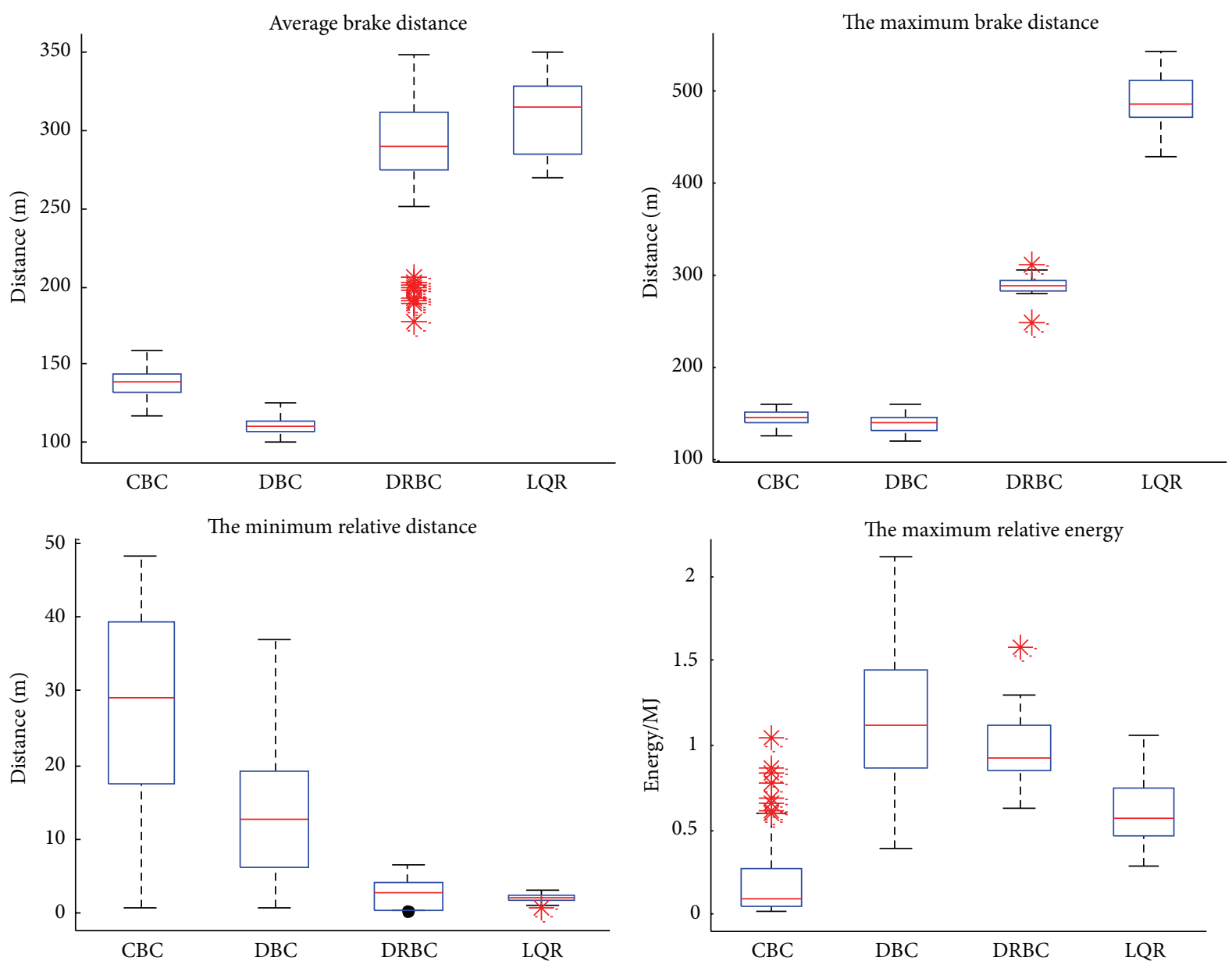

FIGURE 15: Statistical data of brake successful cases using four different control strategies (CBC: 95 cases; DBC: 81 cases; DRBC: 16 cases; LQR: 84 cases).

longitudinal scenarios can be described with some or all the constraints in (5).

The impact (objective function) is defined as the total relative kinetic energy between consecutive pairs of vehicles, which is a sufficient condition for collision impact minimization. MPC is used for control design which ends up with a constrained quadratic programming problem. In the simulation, vehicle masses have been generated randomly, which, in turn, determine vehicle lengths and maximum physical decelerations. If one does not impose minimum deceleration limit for the leader vehicle and the maximum deceleration for the last vehicle in the coupled group, it is likely that most crash could be avoided by braking control. However, in practice, the coupled group could not be completely isolated from other vehicles in the front and behind. Nine vehicles were used for all simulation scenarios.

The refined results will be implemented with experimental vehicles with wireless connection and automatic brake control capabilities. The brake control will be used to track the desired deceleration for each vehicle with the algorithm developed in this paper. The algorithm can be implemented as "centralized" or "decentralized." To be implemented as centralized, one vehicle in the coupled group needs to act as the coordinator to calculate the desired deceleration for each vehicle and pass it over in real time. To be implemented as "decentralized," each vehicle can calculate independently its desired deceleration and activate it since the information of all vehicles is available to each vehicle through V2V. Future work could evaluate which way is more efficient, reliable, and economical in energy use.

\section{Conflict of Interests}

The authors declare that there is no conflict of interests regarding the publication of this paper.

\section{Acknowledgments}

This work was supported by National Natural Science Foundation of China, no. 51175290 and no. 51205228 . The authors would like to thank the support of the Chinese National Programs for High Technology Research and Development, no. 2012AA111901, and the Science Fund of State Key Laboratory of Automotive Safety and Energy, Tsinghua University, no. KF11011. The authors are grateful for Mr. Xiaohui Qing (Tsinghua University) for his comments. 

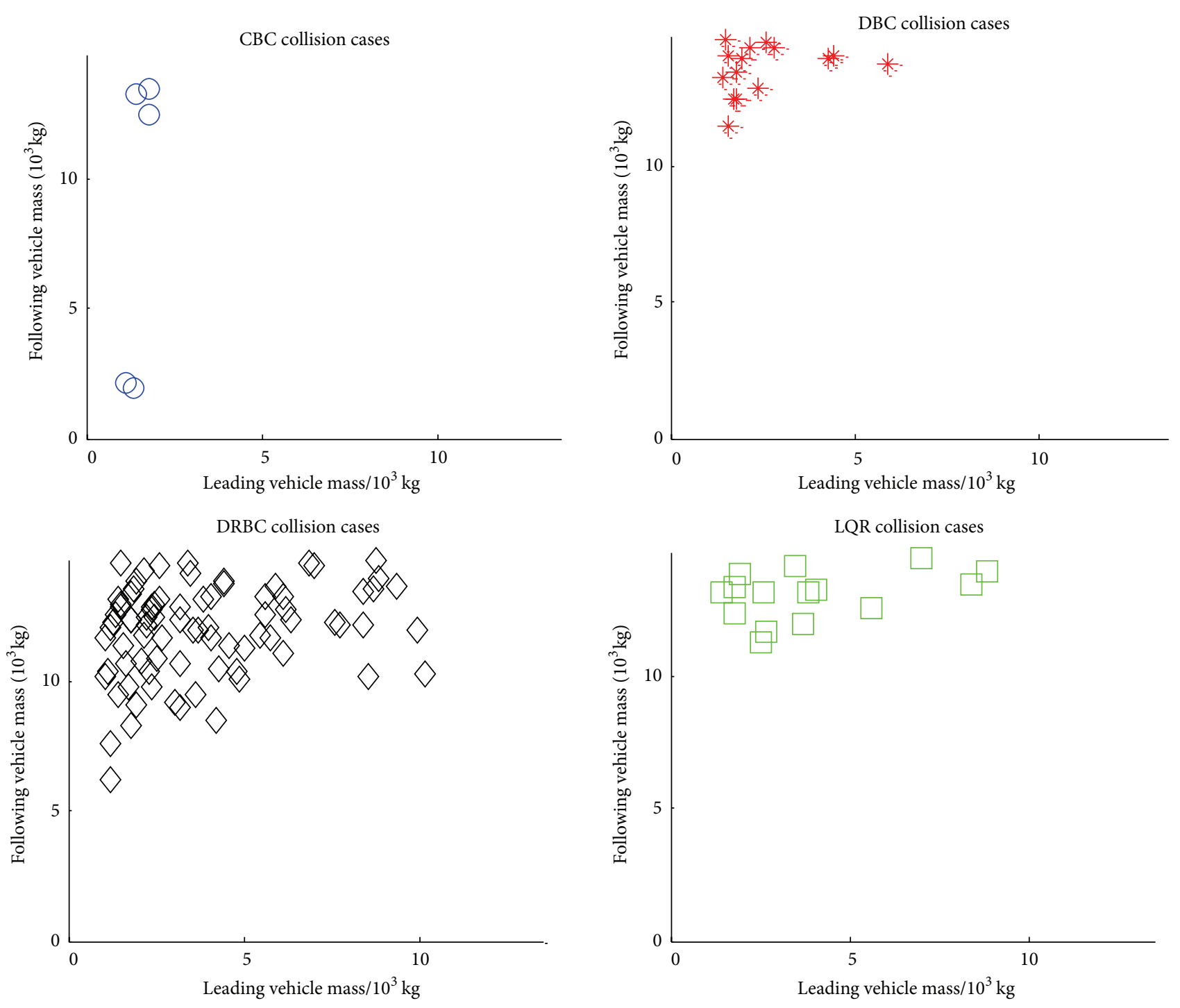

Figure 16: Collision vehicle type.

\section{References}

[1] MPS-DTM: (Ministry of Public Security-Department of Traffic Management), Annual Report of Road Traffic Accidents Statistics in P.R. China, Scientific Research Institute of Traffic Management, Ministry of Public Security, Beijing, China, 2010, (Chinese).

[2] Multiple-vehicle collision real examples, http://en.wikipedia .org/wiki/Multiple-vehicle_collision.

[3] China Multiple Vehicle Crash Examples, http://news.163.com/ 09/1228/11/5RKA5I6F000120GR.html.

[4] P. Fancher, R. Ervin, J. Sayer et al., "Intelligent cruise control field operational test," Final Report DOT HS 808 849, 1998.

[5] S. Li, K. Li, R. Rajamani, and J. Wang, "Model predictive multiple-objective vehicular adaptive cruise control," IEEE Transactions on Control Systems and Technology, vol. 19, no. 3, pp. 556-566, 2011.

[6] X.-Y. Lu and K. Hedrick, "Practical string stability for longitudinal control of automated vehicles," Vehicle System Dynamics, vol. 41, pp. 577-586, 2004.
[7] X.-Y. Lu, J. K. Hedrick, and M. Drew, "ACC/CACC-control design, stability and robust performance," in Proceedings of the American Control Conference, pp. 4327-4332, Anchorage, Alaska, USA, May 2002.

[8] J. Jansson, J. Johansson, and F. Gustafsson, "Decision making for collision avoidance systems," SAE paper, 2002-01-0403.

[9] Precrash, http://en.wikipedia.org/wiki/Precrash_system.

[10] "Honda Develops World's First "Collision Mitigation Brake System” (CMS) for Predicting Rear-end Collisions and Controlling Brake Operations," http://world.honda.com/news/2003/ 4030520.html.

[11] J. Jansson and F. Gustafsson, "A framework and automotive application of collision avoidance decision making," Automatica, vol. 44, no. 9, pp. 2347-2351, 2008.

[12] C. Grover, I. Knight, F. Okoro et al., "Automated emergency brake systems: technical requirements, cost and benefits," Published Project Report (PPR) 227, 2008.

[13] J. K. Hedrick, "Nonlinear controller design for automated vehicle applications," in Proceedings of the UKACC International Conference Control, pp. 23-31, Swansea, UK, September 1998. 
[14] X.-Y. Lu and J. K. Hedrick, "Impact of combined longitudinal, lateral and vertical control on autonomous road vehicle design," International Journal of Vehicle Autonomous Systems, vol. 2, no. 1-2, pp. 40-70, 2004.

[15] H. J. Fang, X. Y. Lu, J. Lu, and Z. Lin, "System optimization in the control of heavy duty vehicle braking sub-systems," in Proceedings of the 48th IEEE Conference on Decision and Control Held Jointly with 28th Chinese Control Conference (CDC/CCC '09), pp. 3563-3568, Shanghai, China, December 2009.

[16] R. N. Dang, F. Zhang, Y. Takae, W. B. Chu, and K. Q. Li, "Braking characteristics of chinese driver in highway and urban road," in Proceedings of the 7th IFAC Symposium on Advances in Automotive Control (AAC '13), pp. 322-327, September 2013.

[17] K. Lee and H. Peng, "Evaluation of automotive forward collision warning and collision avoidance algorithms," Vehicle System Dynamics, vol. 43, no. 10, pp. 735-751, 2005.

[18] F. Morbidi, P. Colaneri, and T. Stanger, "Decentralized optimal control of a car platoon with guaranteed string stability," in Proceedings of the 12th European Control Conference (ECC '13), pp. 3494-3499, IEEE, July 2013.

[19] G. Johansson and K. Rumar, "Drivers' brake reaction times," Human Factors, vol. 13, no. 1, pp. 23-27, 1971. 


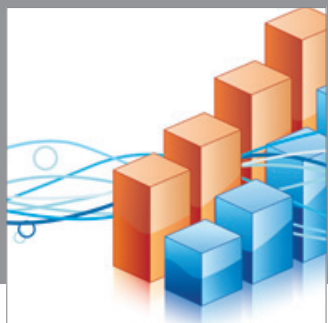

Advances in

Operations Research

mansans

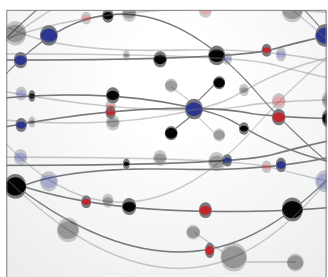

The Scientific World Journal
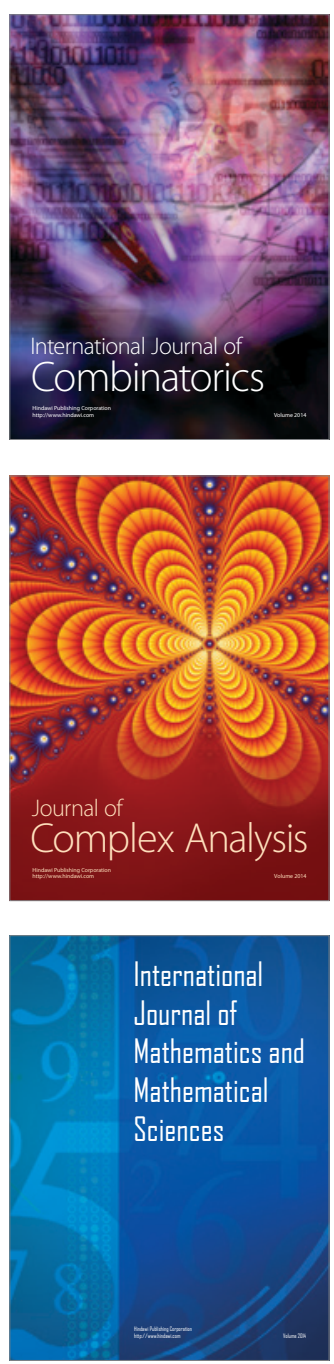
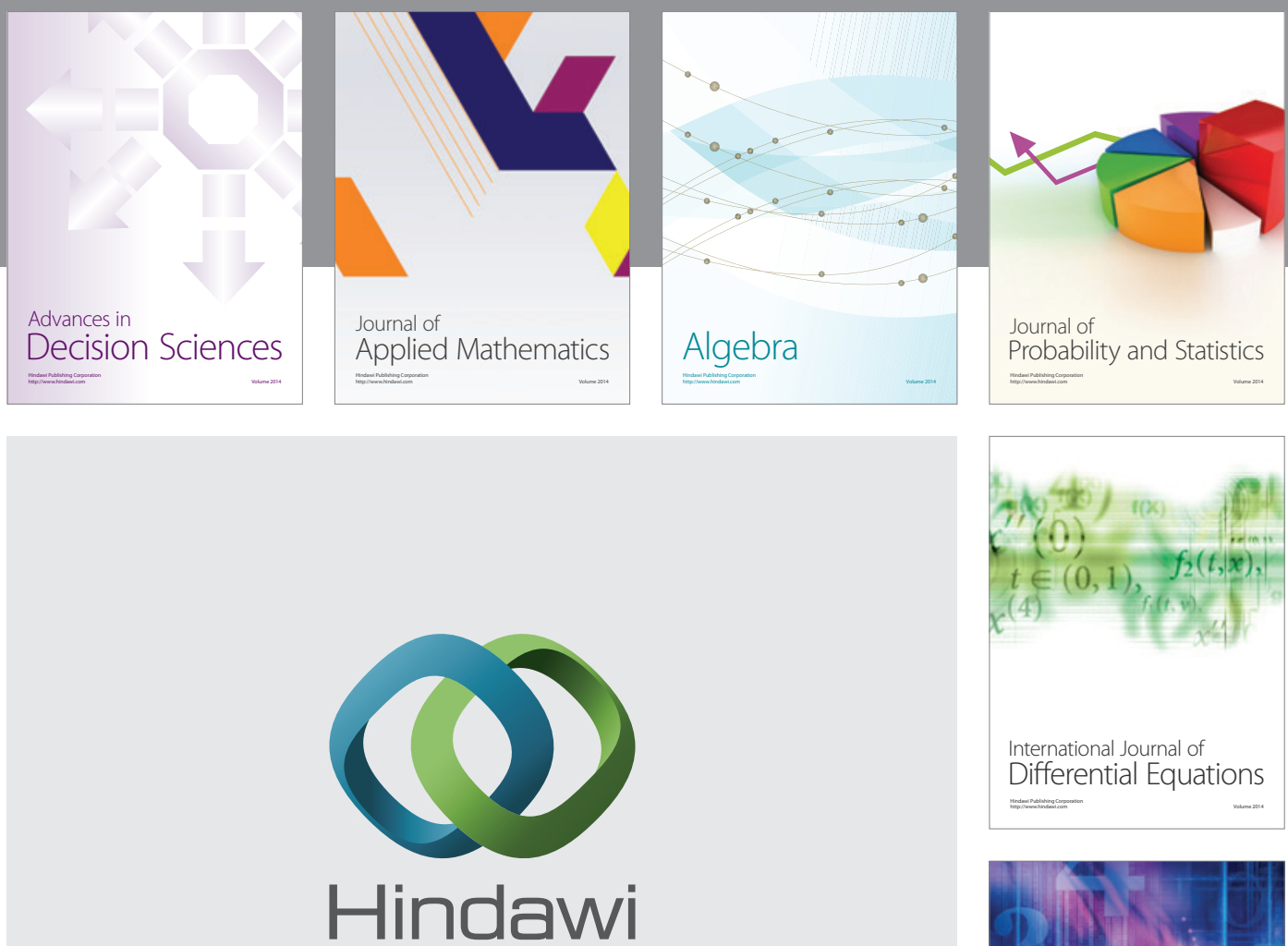

Submit your manuscripts at http://www.hindawi.com
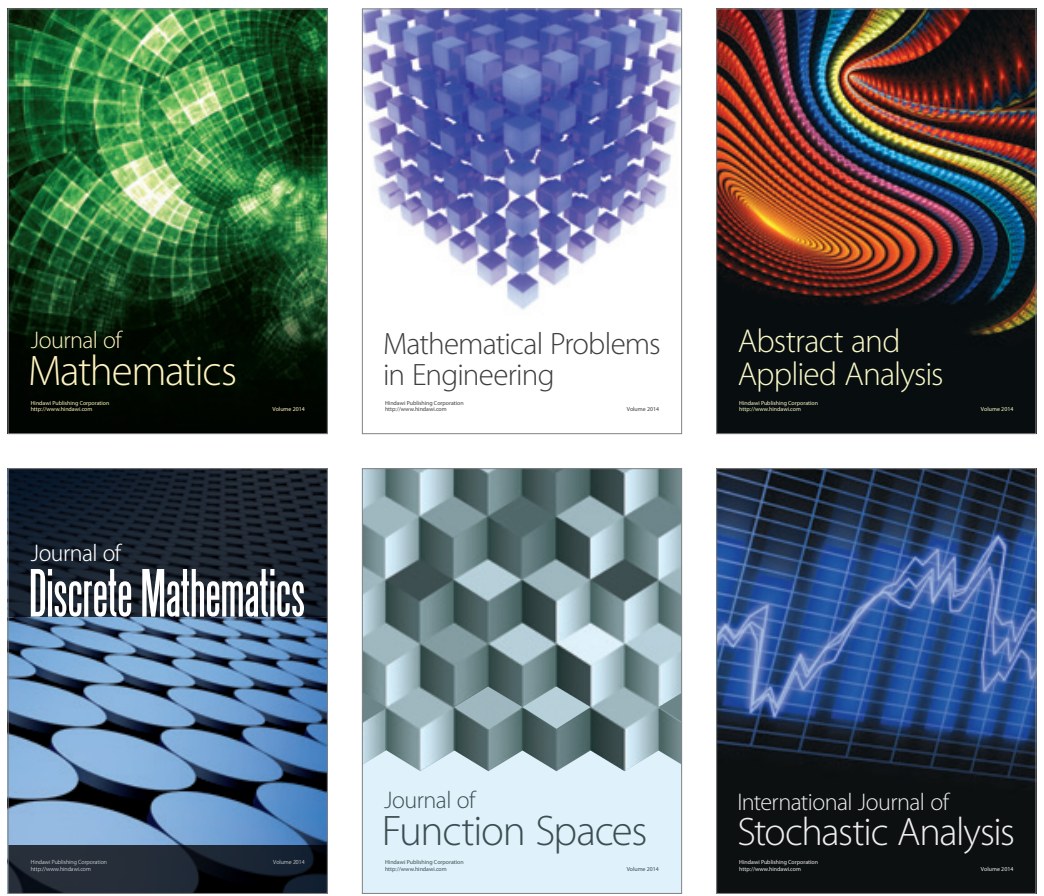

Journal of

Function Spaces

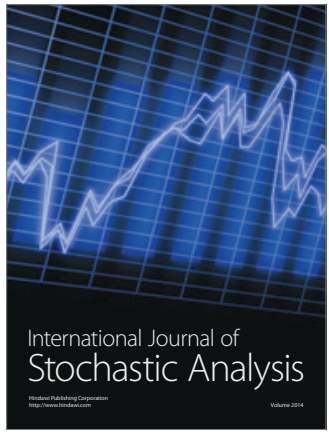

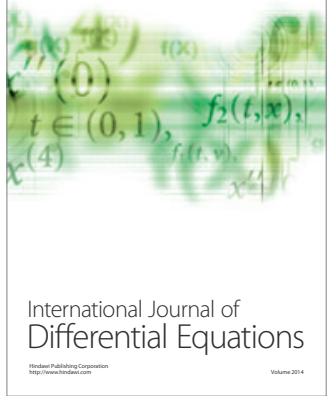
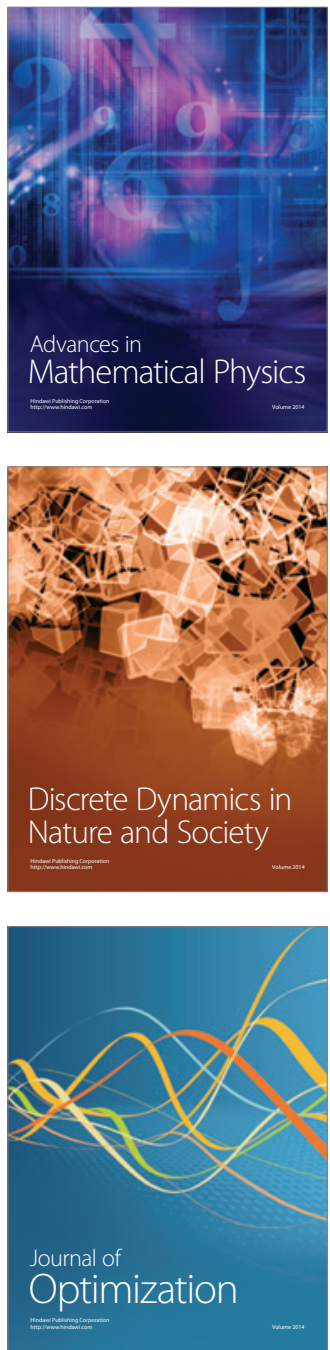